\title{
Introducing the Pair-Angle Distribution Function: many-atom statistics of crystals and disordered materials
}

\author{
A. V. Martin ${ }^{1}$, J. Binns ${ }^{1}$, P. Adams ${ }^{1}$, T. L. Greaves ${ }^{1}$, C. Darmanin ${ }^{2}$ \\ ${ }^{1}$ School of Science, RMIT University, Melbourne, Victoria 3000, Australia, \\ ${ }^{2}$ ARC Centre of Excellence for Advanced Molecular Imaging, La Trobe Institute for Molecular Science, La Trobe University, \\ Melbourne, Victoria, 3086, Australia.
}

andrew.martin@rmit.edu.au

When sample conditions for conventional crystallography are not met (i.e. a large, well-ordered crystal) then x-ray diffraction techniques often do not yield an unambiguous 3D atomic structure. This can occur in powder diffraction and small-angle $\mathrm{x}$-ray scattering (SAXS), where ensembles of crystals or particles in random orientations produce isotropic diffraction around the beam axis. It also occurs for disordered materials, such amorphous solids and liquids, where randomness at the molecular scale has a similar suppression of accessible structural information via x-ray scattering. The accessible structural information is the distribution of atompair distances (known as the pair distribution function or PDF). The PDF has no information about local angular structure, such as bond angles, and in many cases does not uniquely determine the 3D structure.

Fluctuation x-ray scattering (FXS) [1,2] aims to measure the local angular structure in disordered materials using a small x-ray beam to enhance angular scattering fluctuations. We have developed a method of inverting FXS data to recover a sum of three- and fouratom distributions in real-space [3]. We call this 3D function the Pair-Angle Distribution Function (PADF). It is a natural generalisation of the widely used PDF to higher dimensions. The PADF contains, for example, a bond angle distribution and massively increases the amount of structural information beyond that of the PDF.

There are exciting opportunities to combine PADF analysis with crystallography, powder diffraction and SAXS. It could yield new routes to crystal structures, nanoscale disorder, amorphous structure and liquid structure. Here we give an introduction to the PADF and report on our early experimental results with synchrotron, $\mathrm{x}$-ray free-electron lasers and electron microscopes. These include applications to self-assembled lipids [4], disordered carbon materials [5], protein crystals[6] and liquids.

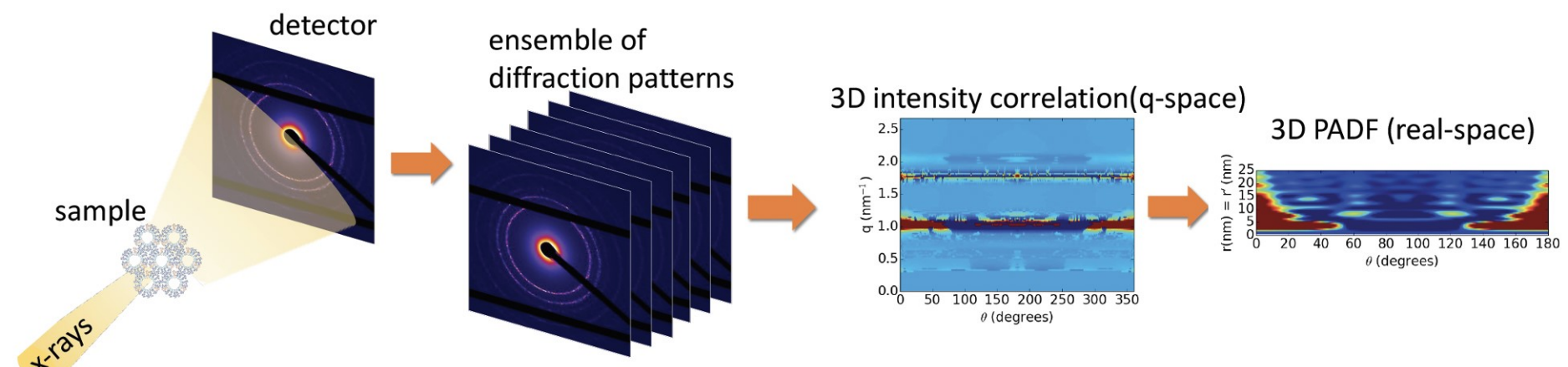

Figure 1. The key steps in PADF analysis. An ensemble of diffraction patterns is measured by translating the sample relative to the beam. A statistical analysis is performed on the ensemble of diffraction patterns (intensity correlation analysis), which is finally converted into real-space to yield the 3D PADF.

[1] Kurta, R.P., Altarelli, M. and Vartanyants, I.A. (2016). "Structural analysis by x-ray intensity angular cross-correlations" in Advances in Chemical Physics (eds S.A. Rice and A.R. Dinner).

[2] Kam, Z. (1977). Macromolecules, 10(5), 927-934.

[3] Martin, A. V. (2017). IUCrJ, 4, 24-36.

[4] Martin, A. V., et al., (2020). Small, 2000828, 1-6

[5] Martin, A. V., et al., (2020). Communications Materials, 1(40), 1-8.

[6] Adams, P.,, et al., (2020). Crystals, 10, 724.

Keywords: Fluctuation x-ray scattering; pair-angle distribution function; SAXS; powder diffraction 